See discussions, stats, and author profiles for this publication at: https://www.researchgate.net/publication/309209279

\title{
Warm-up for Sprint Swimming: Race-Pace or Aerobic Stimulation? A Randomized Study
}

Article in The Journal of Strength and Conditioning Research · October 2016

DOI: $10.1519 /$ JSC. 0000000000001701

CITATIONS

6

7 authors, including:

Henrique P. Neiva

Universidade da Beira Interior

113 PUBLICATIONS 306 CITATIONS

SEE PROFILE

Mikel Izquierdo

Universidad Pública de Navarra

491 PUBLICATIONS 12,396 CITATIONS

SEE PROFILE

Some of the authors of this publication are also working on these related projects:

Project Warm-up and performance View project

Participative, Health and Active Lifestyle in frail institutionalised-dwelling individuals: PHALIF study, a research arm of PRO-HMESCI View project
READS

1,288

Mário C Marques

Universidade da Beira Interior

279 PUBLICATIONS 2,588 CITATIONS

SEE PROFILE

João L Viana

Instituto Superior da Maia

100 PUBLICATIONS 781 CITATIONS

SEE PROFILE 


\title{
Warm-up for Sprint Swimming: RACe-Pace or Aerobic Stimulation? A Randomized Study
}

\author{
Henrique P. Neiva, ${ }^{1,2}$ Mário C. Marques, ${ }^{1,2}$ Tiago M. Barbosa, ${ }^{2,3}$ Mikel Izquierdo, ${ }^{4}$ \\ João L. Viana, ${ }^{2,5}$ Ana M. Teixeira, ${ }^{6}$ and Daniel A. Marinho ${ }^{1,2}$ \\ ${ }^{1}$ Department of Sport Sciences, University of Beira Interior, Covilhã, Portugal; ${ }^{2}$ Research Center in Sport Sciences, Health \\ Sciences and Human Development, CIDESD, Covilhã, Portugal; ${ }^{3}$ National Institute of Education, Nanyang Technological \\ University, Singapore; ${ }^{4}$ Department of Health Sciences, Public University of Navarre, Navarre, Spain; ${ }^{5}$ University Institute of \\ Maia, ISMAI, Maia, Portugal; and ${ }^{6}$ Faculty of Sport Sciences and Physical Education, Coimbra, Portugal
}

\begin{abstract}
Neiva, HP, Marques, MC, Barbosa, TM, Izquierdo, M, Viana, JL, Teixeira, AM, and Marinho, DA. Warm-up for sprint swimming: race-pace or aerobic stimulation? A randomized study. J Strength Cond Res 31(9): 2423-2431, 2017-The aim of this study was to compare the effects of 2 different warm-up intensities on 100-m swimming performance in a randomized controlled trial. Thirteen competitive swimmers performed two 100-m freestyle time-trials on separate days after either control or experimental warm-up in a randomized design. The control warm-up included a typical race-pace set $(4 \times 25 \mathrm{~m})$, whereas the experimental warm-up included an aerobic set $(8 \times 50 \mathrm{~m}$ at 98-102\% of critical velocity). Cortisol, testosterone, blood lactate $\left(\left[\mathrm{La}^{-}\right]\right)$, oxygen uptake $\left(\dot{\mathrm{V}}_{2}\right)$, heart rate, core (Tcore and Tcore $_{\text {net }}$ ) and tympanic temperatures, and rating of perceived exertion (RPE) were monitored. Stroke length (SL), stroke frequency (SF), stroke index (SI), and propelling efficiency $\left(\eta_{p}\right)$ were assessed for each 50-m lap. We found that $\dot{\mathrm{V}}_{2}$, heart rate, and Tcore $_{\text {net }}$ were higher after experimental warm-up ( $d>$ 0.73), but only the positive effect for Tcore net $_{\text {was }}$ maintained until the trial. Performance was not different between conditions $(d=0.07)$. Experimental warm-up was found to slow SF (mean change $\pm 90 \% \mathrm{CL}=2.06 \pm 1.48 \%$ ) and increase SL $(1.65 \pm 1.40 \%)$ and $\eta_{p}(1.87 \pm 1.33 \%)$ in the first lap. After the time-trials, this warm-up had a positive effect on Tcore $_{\text {net }}$ $(d=0.69)$ and a negative effect on $\left[\mathrm{La}^{-}\right](d=0.56)$. Although the warm-ups had similar outcomes in the $100-\mathrm{m}$ freestyle, performance was achieved through different biomechanical strategies. Stroke length and efficiency were higher in the first lap after the experimental warm-up, whereas SF was higher after control warm-up. Physiological adaptations were observed mainly through an increased Tcore after experimental
\end{abstract}

Address correspondence to Mikel Izquierdo, mikel.izquierdo@gmail.com. $31(9) / 2423-2431$

Journal of Strength and Conditioning Research

(c) 2016 National Strength and Conditioning Association warm-up. In this condition, the lower $\left[\mathrm{La}^{-}\right]$after the trial suggests lower dependency on anaerobic metabolism.

KEY WoRDS performance, pre-exercise, intensities,
physiology, biomechanics

\section{INTRODUCTION}

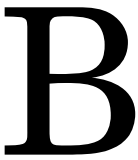

efore a competitive event, swimmers usually engage in different activities to change their physiological status to optimize performance (20-22). These activities are intended to increase body temperature, resulting in physiological changes such as increased muscle efficiency, increased blood flow, improved efficiency of muscle glycolysis and high-energy phosphate degradation during exercise, and an increased nerve conduction rate $(5,20)$. To achieve these measures, different routines are recommended before a swim race event, despite little scientific evidence supporting which changes result from the structure of the warm-up $(13,23)$.

Previous research has investigated the effect of different durations of in-water warm-up $(2,25)$ and recovery time after warm-up $(24,34,37)$, but few studies have focused on the effects of different warm-up intensities on subsequent swimming performance $(2,13)$. One relevant study on the intensity of priming exercitation in swimming was conducted by Houmard et al. (13). The authors looked at 2 different warm-up intensities, comparing the effects of continuous swimming for a distance of $365.8 \mathrm{~m}$ at $\sim 65 \%$ of peak oxygen uptake $\left(\dot{\mathrm{V}}_{2}\right.$ peak $)$ with intermittent swimming at $\sim 95 \% \dot{\mathrm{V}}_{2}$ peak, while keeping the other variables (i.e., the distance swam) the same. No differences were observed in heart rate, stroke length (SL), or blood lactate concentrations $\left(\left[\mathrm{La}^{-}\right]\right)$after the trial. These results suggested that there is no benefit to designing high-intensity sets for a warm-up. Nevertheless, it is common to include racepace sets in the prerace warm-up (21), which may have been influenced by anecdotal suggestions that race-pace warmups increase preparedness (22).

With regard to this topic, to date, only submaximal trials have been evaluated after warm-up, or the total distance has 
TABLE 1. Warm-up protocols.

\section{Warm-up Task}

\begin{tabular}{ccc}
\hline 1 & $300 \mathrm{~m}(100-\mathrm{m}$ usual breathing, $100-\mathrm{m}$ breathing in the fifth stroke, $100-\mathrm{m}$ usual breathing) \\
2 & $4 \times 100 \mathrm{~m}$ on $1: 50(2 \times[25 \mathrm{~m}$ kick $+25 \mathrm{~m}$ increased stroke length $])$ \\
3 & Control: $8 \times 50 \mathrm{~m}$ on $1: 00(2 \times 50 \mathrm{~m}$ drill; $2 \times 50 \mathrm{~m}$ building up velocity; Experimental: $8 \times 50 \mathrm{~m}$ on $1: 00$ (98- \\
4 & $4 \times[25 \mathrm{~m}$ race pace $+25 \mathrm{~m}$ easy $])$ & $102 \%$ of critical velocity) \\
4 & $100 \mathrm{~m}$ (easy swim) & $100 \mathrm{~m}$ (easy swim) \\
\hline
\end{tabular}

not been the same between warm-ups being compared; furthermore, no clear evidence regarding specific intensities has been reported (2). An interesting approach to the issue of warm-up intensity was recently taken by Wittekind and Beneke (36) for cycling. The authors found that the reduced anaerobic glycolytic contribution during 1 minute of cycling on an ergometer after a higher intensity warm-up may be compensated for by increased aerobic stimulation. In support of this, some authors have previously reported that warm-up may optimize performance by enhancing oxygen uptake $\left(\dot{\mathrm{VO}}_{2}\right)$ kinetics $(14,19,32)$. Faster $\dot{\mathrm{VO}}_{2}$ and the consequent reduction in anaerobic glycolytic contribution could delay anaerobic metabolism and perhaps reduce metabolic fatigue. Such $\dot{\mathrm{V}}_{2}$ stimulation may be of particular interest when applied to sprinting events, such as the $100-\mathrm{m}$ swimming race, in place of traditional race-pace sets; yet, no evidence for this has been reported in the literature. Despite the short duration of the $100-\mathrm{m}$ race, both metabolic energy systems (anaerobic and aerobic) contribute approximately $50 \%$ of the total energy required, highlighting the relevance of both (28).

It is critical to understand how different warm-up intensities influence swimming performance and swimmers' physiological, biomechanical, and psychophysiological responses. Despite the known impact of different warm-up intensities in other sports $(8,36)$, little information is available for one of the most popular Olympic sports, swimming, and one of its main events, the $100-\mathrm{m}$ freestyle. Without scientific evidence, coaches are still mandating at least one set of race-pace efforts be performed during the pool warm-up $(20,21)$, even though it is known that $\dot{\mathrm{V}}_{2}$ could exert a great influence during the swim race (28). Thus, the current study was conducted to compare the effects of 2 different warm-up set intensities on performance, one simulating race-pace and the other targeting $\dot{\mathrm{V}} \mathrm{O}_{2}$. To gain a deeper understanding of the mechanisms explaining the acute response, biomechanical, physiological, and psychophysiological adaptations were also assessed. It was hypothesized that performance would improve when race-pace sets were included in the warmup routine because of the stimulation of metabolic energy pathways recruited during the race. Varying warm-up intensities would result in different biomechanical strategies and physiological/psychophysiological responses.

\section{Methods}

\section{Experimental Approach to the Problem}

The purpose of the current study was to evaluate the effects of using a race-pace or an aerobic stimulation swimming set during warm-up on the $100-\mathrm{m}$ freestyle, in high-level swimmers, in terms of performance, and biomechanical, physiological and psychophysiological response. The study followed a repeated measures design.

Each participant completed 2 time-trials of the $100-\mathrm{m}$ freestyle, one after the experimental warm-up condition and one after the control warm-up, in randomized order, separated by 48 hours. The dependent variables were time, kinetics, and kinematics during the $100-\mathrm{m}$ trial; the metabolic, cardiovascular, psychophysiological, temperature, and hormonal variables during warm-up; transition phase between warm-up and time-trial; and recovery period after trial. This design was able to test whether the warm-ups using a race-pace set or aerobic stimulation set affected

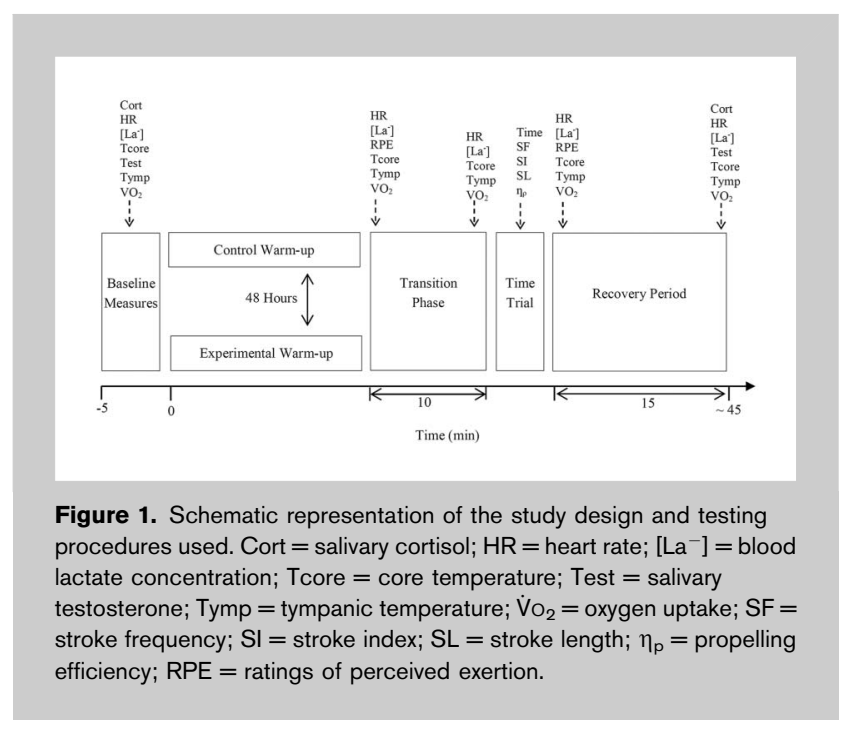


TABLE 2. Mean $\pm S D$ values of physiological and psychophysiological variables assessed after warm-up (post) and before trial (pretrial) during control (CWU) and experimental (WU) procedures $(n=13)$.*

\begin{tabular}{|c|c|c|c|c|c|c|c|}
\hline & CWU & WU & $p$ & $d$ & $\begin{array}{c}\text { Mean } \% \\
\text { change; } \pm 90 \% \\
\mathrm{CL} \dagger\end{array}$ & $\begin{array}{c}\% \\
\text { Chance } \ddagger\end{array}$ & $\begin{array}{l}\text { Qualitative } \\
\text { inference }\end{array}$ \\
\hline \multicolumn{8}{|c|}{$\begin{array}{l}\dot{\mathrm{V}}_{2} \\
\quad\left(\mathrm{ml} \cdot \mathrm{kg}^{-1} \cdot \mathrm{min}^{-1}\right)\end{array}$} \\
\hline Post§ & $18.15 \pm 5.40$ & $22.84 \pm 5.15$ & 0.03 & 0.73 & $28.4 \pm 25.7$ & $95 / 4 / 1$ & Very likely +ive \\
\hline Pretrial & $6.58 \pm 1.29$ & $6.71 \pm 1.45$ & 0.66 & 0.13 & $1.7 \pm 8.3$ & $32 / 59 / 10$ & Unclear \\
\hline \multicolumn{8}{|c|}{$\begin{array}{l}\text { Heart Rate } \\
\qquad\left(b \cdot \min ^{-1}\right)\end{array}$} \\
\hline Post $\|$ & $93 \pm 13$ & $107 \pm 12$ & $<0.01$ & 1.73 & $15.7 \pm 5.4$ & $100 / 0 / 0$ & Most likely +ive \\
\hline $\begin{array}{c}\text { Pretrial } \\
{\left[\mathrm{La}^{-}\right](\mathrm{mm}}\end{array}$ & $95 \pm 11$ & $97 \pm 10$ & 0.66 & 0.13 & $1.9 \pm 7.3$ & $43 / 42 / 15$ & Unclear \\
\hline Post & $3.87 \pm 1.01$ & $3.96 \pm 2.23$ & 0.88 & 0.04 & $-11.1 \pm 27.9$ & $42 / 27 / 31$ & Unclear \\
\hline Pretrial & $2.88 \pm 0.78$ & $3.01 \pm 1.36$ & 0.68 & 0.11 & $-2.9 \pm 24.2$ & $47 / 35 / 19$ & Unclear \\
\hline \multicolumn{8}{|c|}{ Tcore $\left({ }^{\circ} \mathrm{C}\right)$} \\
\hline Post & $37.66 \pm 0.28$ & $37.91 \pm 0.30$ & 0.06 & 0.74 & $0.7 \pm 0.6$ & $93 / 5 / 1$ & Likely +ive \\
\hline Pretrial & $37.61 \pm 0.29$ & $37.80 \pm 0.35$ & 0.28 & 0.40 & $0.5 \pm 0.8$ & $76 / 16 / 8$ & Unclear \\
\hline \multicolumn{8}{|c|}{ Tcore $_{\text {net }}\left({ }^{\circ} \mathrm{C}\right)$} \\
\hline Post $\|$ & $0.43 \pm 0.28$ & $0.62 \pm 0.32$ & 0.01 & 1.10 & $71.5 \pm 76.1$ & $97 / 3 / 0$ & Very likely +ive \\
\hline Pretrial & $0.36 \pm 0.33$ & $0.51 \pm 0.38$ & 0.13 & 0.56 & $21.1 \pm 125.2$ & $80 / 18 / 2$ & Likely +ive \\
\hline \multicolumn{8}{|c|}{ Tympanic $\mathrm{T}\left({ }^{\circ} \mathrm{C}\right)$} \\
\hline Post & $34.15 \pm 0.32$ & $33.96 \pm 0.63$ & 0.28 & 0.32 & $-0.5 \pm 0.9$ & $7 / 17 / 76$ & Unclear \\
\hline $\begin{array}{l}\text { Pretrial } \\
\text { RPE }\end{array}$ & $35.67 \pm 0.72$ & $35.88 \pm 0.47$ & 0.17 & 0.40 & $0.6 \pm 0.7$ & $64 / 34 / 1$ & Possibly +ive \\
\hline Post $\|$ & $12.92 \pm 1.55$ & $13.92 \pm 1.75$ & $<0.01$ & 0.87 & $7.5 \pm 4.8$ & $97 / 3 / 0$ & Very likely +ive \\
\hline
\end{tabular}

${ }^{*} 90 \% \mathrm{CL}=90 \%$ confidence limits; $\mathrm{V}_{2}=$ oxygen uptake; + ive, - ive $=$ positive and negative changes, respectively; $\left[\mathrm{La}^{-}\right]=$blood lactate concentration; Tcore $=$ core temperature; Tcore $_{n e t}=$ core temperature net values; Tympanic $\mathrm{T}=$ tympanic temperature. $\dagger$ Positive \% change equates to an increase in WU condition. $\ddagger$ Presented as harmful/trivial/beneficial for performance (time) and positive/trivial/negative for other variables. $\S p \leq 0.05$. $\| p \leq 0.01$.

swimming performance (independent variables). The investigation protocol consisted of 2 testing sessions, scheduled on different days (with 2 conditions assessed for each participant).

\section{Subjects}

Swimmers were eligible for the study if they had competed at the Portuguese national level for the last 6 years. Thirteen competitive male swimmers aged $15-20$ years (mean $\pm S D$ : $17.15 \pm 1.52$ years of age, $1.77 \pm 0.07 \mathrm{~m}$ height, $64.80 \pm 8.58$ $\mathrm{kg}$ body mass, $8.20 \pm 1.52$ years of training background) were recruited. All swimmers had previously competed in national swimming championship finals and had completed different warm-ups over the previous years. The average personal best time in the $100-\mathrm{m}$ freestyle was $56.79 \pm 2.24 \mathrm{~s}$ (567.85 \pm 66.79 FINA 2015 scoring points). After local ethics board approval, ensuring compliance with the Declaration of Helsinki, the participants were informed about the study procedures, and a written informed consent was obtained from the subjects or the parent or guardian when subjects were under 18 years of age.

\section{Procedures}

All the procedures took place at the same time of the day (8:00-12:00 AM) in a 50-m indoor swimming pool with a water temperature of $28.12 \pm 0.09^{\circ} \mathrm{C}$, air temperature of $27.95 \pm 0.16^{\circ} \mathrm{C}$, and humidity of $60.20 \pm 0.58 \%$. The swimmers were familiarized with the warm-up procedures 48 hours before the experiments, and they were reminded to maintain the same training, recovery, and diet routines during the assessment days, avoiding strenuous exercise, and abstaining from smoking and consuming caffeine 48 hours before testing. Upon arrival at the pool, the swimmers remained seated for 5 minutes to assess baseline measurements of cortisol, testosterone, heart rate (Vantage NV; Polar, Kempele, Finland), tympanic temperature (Thermoscan IRT 4520; Braun, Kronberg, Germany), core temperature (Tcore; CorTemp; HQ Inc., Palmetto, FL, USA), [La ${ }^{-}$(Accutrend Lactate; Roche, Mannheim, Germany), and $\dot{\mathrm{VO}}_{2}$ (K4b2; Cosmed, Rome, Italy). Each swimmer was then randomly assigned to a warm-up protocol (Table 1 and Figure 1).

Both warm-ups involved the recommended swim distance of $1,200 \mathrm{~m}$ (25), with the difference between protocols 
TABLE 3. Mean $\pm S D$ values of the $100-\mathrm{m}$ and $50-\mathrm{m}$ lap times, biomechanical, physiological, and psychophysiological variables assessed during control (CWU) and experimental (WU) procedures $(n=13)$. *

\begin{tabular}{|c|c|c|c|c|c|c|c|}
\hline & CWU & WU & $p$ & $d$ & $\begin{array}{c}\text { Mean } \% \\
\text { change; } \pm 90 \% \\
\text { CL } \dagger\end{array}$ & $\begin{array}{c}\% \\
\text { Chance } \ddagger\end{array}$ & $\begin{array}{l}\text { Qualitative } \\
\text { inference }\end{array}$ \\
\hline $100 \mathrm{~m}(\mathrm{~s})$ & $57.87 \pm 1.84$ & $57.83 \pm 1.77$ & 0.79 & 0.07 & $-0.1 \pm 0.5$ & $0 / 99 / 1$ & Very likely trivial \\
\hline 1 st $50 \mathrm{~m}$ (s) & $27.67 \pm 0.99$ & $27.70 \pm 0.95$ & 0.30 & 0.31 & $0.4 \pm 0.7$ & $19 / 80 / 0$ & Likely trivial \\
\hline 2nd 50 m (s) & $30.31 \pm 1.05$ & $30.13 \pm 0.92$ & 0.12 & 0.48 & $-0.6 \pm 0.6$ & $0 / 73 / 27$ & $\begin{array}{l}\text { Possibly } \\
\text { beneficial }\end{array}$ \\
\hline $1 \mathrm{st} 15 \mathrm{~m}(\mathrm{~s})$ & $6.74 \pm 0.28$ & $6.76 \pm 0.29$ & 0.56 & 0.09 & $0.3 \pm 0.8$ & $14 / 84 / 2$ & Likely trivial \\
\hline 1st 50 m SF $(\mathrm{Hz}) \S$ & $0.90 \pm 0.07$ & $0.88 \pm 0.06$ & 0.03 & 0.74 & $2.1 \pm 1.5$ & $0 / 30 / 70$ & Possibly -ive \\
\hline 2nd 50 m SF $(\mathrm{Hz})$ & $0.76 \pm 0.06$ & $0.77 \pm 0.06$ & 0.48 & 0.30 & $0.8 \pm 2.2$ & $30 / 67 / 03$ & Possibly +ive \\
\hline 1st $50 \mathrm{~m} \mathrm{SL}(\mathrm{m}) \S$ & $2.04 \pm 0.15$ & $2.07 \pm 0.14$ & 0.05 & 0.57 & $1.6 \pm 1.4$ & $48 / 52 / 0$ & Possibly +ive \\
\hline 2nd $50 \mathrm{~m} \mathrm{SL}(\mathrm{m})$ & $2.17 \pm 0.14$ & $2.16 \pm 0.15$ & 0.63 & 0.12 & $-0.6 \pm 2.0$ & $5 / 72 / 23 /$ & Unlikely -ive \\
\hline $\begin{array}{l}\text { 1st } 50 \mathrm{~m} \mathrm{SI} \\
\qquad\left(\mathrm{m}^{2} \cdot \mathrm{c}^{-1} \cdot \mathrm{s}^{-1}\right)\end{array}$ & $3.70 \pm 0.33$ & $3.75 \pm 0.31$ & 0.12 & 0.45 & $1.4 \pm 1.5$ & $27 / 73 / 0$ & Possibly +ive \\
\hline $\begin{array}{l}\text { 2nd } 50 \mathrm{~m} \mathrm{SI} \\
\left(\mathrm{m}^{2} \cdot \mathrm{c}^{-1} \cdot \mathrm{s}^{-1}\right)\end{array}$ & $3.56 \pm 0.25$ & $3.54 \pm 0.25$ & 0.71 & 0.15 & $-0.4 \pm 1.9$ & $4 / 81 / 15$ & Likely trivial \\
\hline 1st $50 \mathrm{~m} \eta_{\mathrm{p}}(\%) \S$ & $33.51 \pm 2.68$ & $34.11 \pm 2.35$ & 0.03 & 0.70 & $1.9 \pm 1.3$ & $55 / 45 / 0$ & Possibly +ive \\
\hline 2nd $50 \mathrm{~m} \eta_{\mathrm{p}}(\%)$ & $35.82 \pm 2.89$ & $35.63 \pm 3.16$ & 0.63 & 0.13 & $-0.6 \pm 2.0$ & $3 / 82 / 15$ & Likely trivial \\
\hline $\begin{array}{l}\dot{\mathrm{V}}_{2} \text { peak } \\
\quad\left(\mathrm{mL} \cdot \mathrm{kg}^{-1} \cdot \mathrm{min}^{-1}\right)\end{array}$ & $50.11 \pm 5.79$ & $50.95 \pm 7.41$ & 0.63 & 0.15 & $1.3 \pm 5.9$ & $40 / 48 / 12$ & Unclear \\
\hline Heart Rate $\left(b \cdot \min ^{-1}\right)$ & $160 \pm 15$ & $163 \pm 12$ & 0.21 & 0.50 & $1.8 \pm 4.5$ & $46 / 46 / 8$ & Unclear \\
\hline$\left[\mathrm{La}^{-}\right]_{\text {peak }}\left(\mathrm{mmol} \cdot \mathrm{L}^{-1}\right)$ & $12.60 \pm 2.50$ & $11.58 \pm 3.11$ & 0.07 & 0.56 & $-10.1 \pm 9.2$ & $0 / 17 / 82$ & Likely -ive \\
\hline Tcore $\left({ }^{\circ} \mathrm{C}\right)$ & $37.50 \pm 0.32$ & $37.71 \pm 0.35$ & 0.27 & 0.42 & $0.5 \pm 0.9$ & $77 / 16 / 8$ & Unclear \\
\hline Tcore $_{\text {net }}\left({ }^{\circ} \mathrm{C}\right)$ & $0.19 \pm 0.35$ & $0.36 \pm 0.38$ & 0.09 & 0.69 & $-20.5 \pm 115.0$ & $84 / 14 / 1$ & Likely +ive \\
\hline Tympanic $\mathrm{T}\left({ }^{\circ} \mathrm{C}\right)$ & $34.27 \pm 0.28$ & $34.38 \pm 0.40$ & 0.47 & 0.20 & $0.3 \pm 0.8$ & $63 / 23 / 14$ & Unclear \\
\hline RPE $\|$ & $18.00 \pm 1.29$ & $18.54 \pm 1.20$ & 0.01 & 0.82 & $3.0 \pm 1.8$ & $91 / 9 / 0$ & Likely +ive \\
\hline \multicolumn{8}{|c|}{ 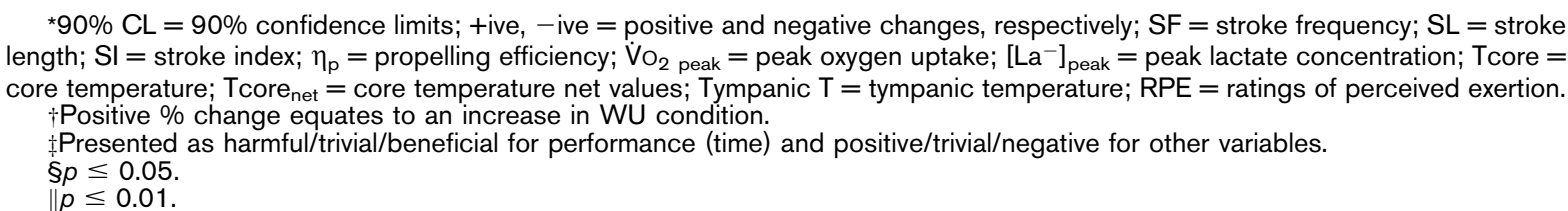 } \\
\hline
\end{tabular}

being the intensity of the main set. Swimmers' warm-up usually includes a race-pace-specific set $(20,21)$. Nevertheless, it is not known whether a $\dot{\mathrm{V}}_{2}$ stimulation set allows for a faster oxygen uptake and perhaps reduces the anaerobic contribution during the race $(14,19)$. Therefore, the control warm-up included a short-distance race-pace set that is usual among swimmers $(20,21)$; whereas during the experimental warm-up, the race-pace set used was aimed at increasing the $\dot{V}_{2}$. The experimental warm-up's main set was structured based on the assumptions that (a) critical velocity can be $3-10 \%$ faster than the velocity at lactate threshold and will lead to a progressive increase in $\dot{\mathrm{V}}_{2}$ (30); (b) short-duration intermittent aerobic sets result in less glycogen depletion compared with continuous sets (4); and (c) $\left[\mathrm{La}^{-}\right]$levels should be lower than $5 \mathrm{mmol} \cdot \mathrm{L}^{-1}$ (16). Critical velocity was calculated from the slope of the regression line between distance and time, combining the 50 and the $400 \mathrm{~m}$ best times (30), and a range between 98 and $102 \%$ was set for pacing the swimmers.
Time-Trial Performance. Once the swimmers finished warming-up, they remained seated for 10 minutes before performing the time-trial. An official start was used, and the times were measured by a timing system (Omega SA, Corgémont, Switzerland), using a stopwatch held by a swimming coach as a backup, and a video camera (Casio Exilim Ex-F1, $f=30 \mathrm{~Hz}$ ) placed at $15 \mathrm{~m}$, perpendicular to lane 7 . These same procedures and devices were also used to assess the $15-\mathrm{m}$ time.

Kinematics and Efficiency. Stroke frequency (SF), SL, stroke index $(\mathrm{SI})$, and propelling efficiency $\left(\eta_{\mathrm{p}}\right)$ were determined according to the procedures used by Neiva et al. (25).

Metabolic, Cardiovascular, and Psychophysiological Variables. Capillary blood samples for $\left[\mathrm{La}^{-}\right]$assessment were collected from the fingertips after warm-up ( 1 minute), immediately before the trial, 3 and 6 minutes after the trial to obtain the highest value $\left(\left[\mathrm{La}^{-}\right]_{\text {peak }}\right)$, and 15 minutes after the trial. 


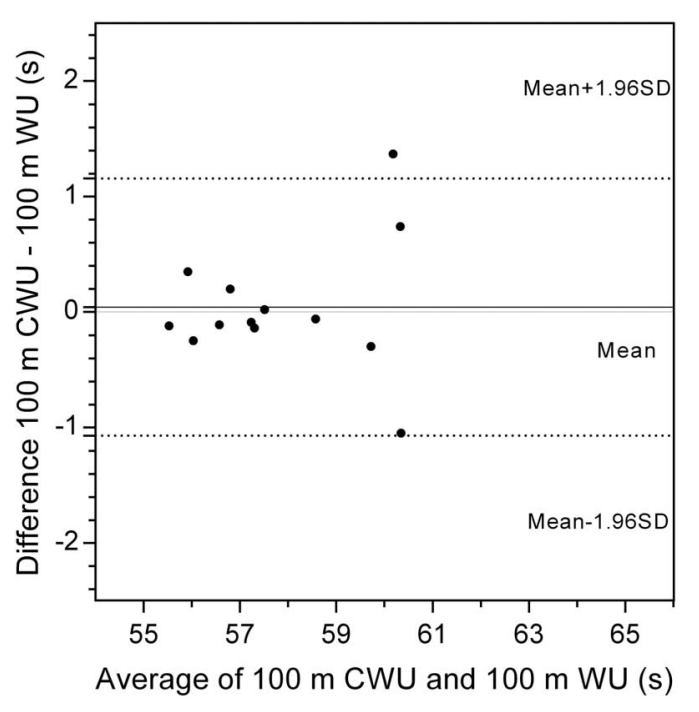

Figure 2. Bland-Altman plots representing the $100-\mathrm{m}$ time in the 2 trial conditions: control warm-up (CWU) and experimental warm-up (WU). Average difference line (solid line) and 95\% confidence limits (dashed lines) are indicated $(n=13)$.

Heart rate was assessed before, during, and after each warmup (first minute) before the trial (ninth minute) and recovery after the time-trial (every minute). Additionally, perceived exertion (RPE) ratings were recorded using a 6-20 Borg scale (7) during and after the warm-up and after trial. $\dot{\mathrm{V}}_{2}$ was monitored during the post warm-up time period and after the $100 \mathrm{~m}$ for 15 minutes. After the trial, the first 2 seconds of measurement after detection were not considered because of adaptation of the device to the sudden change of respiratory cycles and $\dot{\mathrm{V}}_{2}(18)$. The $\dot{\mathrm{V}}_{2}$ peak was considered to be the mean value for the following 6 seconds (18).

Temperature. Each swimmer's tympanic temperature was recorded before and after the warm-up and before and after the trial ( 1 minute). Tcore was assessed by a temperature sensor that was ingested 10 hours before the test (9). This pill transmitted a radio signal to an external sensor, which subsequently converted the signal into digital format. The net values (difference between the values recorded and baseline values) of Tcore ( Tcore $_{\text {net }}$ ) were selected to compare data and reduce error because of pill position.

Hormonal Variables. Saliva samples were collected before (baseline) and after finishing the protocol (15 minutes after the trial). Cortisol and testosterone concentrations were determined by enzyme-linked immunosorbent assays using commercially available kits (Salimetrics, State College, PA, USA). While seated and leaning forward, the participants provided saliva samples using the passive drool method. Samples were collected directly through a plastic drinking straw into 10 -ml plastic screw top tubes; all samples were kept cold immediately after collection $\left(2^{\circ} \mathrm{C}\right)$ and then frozen $\left(-20^{\circ} \mathrm{C}\right)$ until assayed. Each collection tube was identified with numbers and letters corresponding to the participant, procedure, and collection point. The minimum collection time was 3 minutes for each subject to allow for the collection of a sufficient sample volume. No drinking was allowed, and procedures were conducted at the same time of day to avoid circadian influences on performance. The mean intraassay and interassay coefficients of variation were 3.72 and $9.41 \%$ for cortisol and 3.15 and $7.26 \%$ for testosterone, respectively.

\section{Statistical Analyses}

The normality of all distributions was verified using ShapiroWilks tests, and parametric statistical analysis was adopted. To compare the 2 trials, Student's paired $t$-tests and Cohen's $d$ effect sizes were calculated $(p \leq 0.05)$. An effect size of 0.2 was deemed small, 0.5 medium, and 0.8 large. The smallest worthwhile effects were also computed to determine the likelihood that the true effect was substantially beneficial (positive), trivial, or harmful (negative). Magnitude-based inferences were categorized as clinical for performance measures and mechanistic for other measures. The threshold value for smallest worthwhile change was set at $0.8 \%$ for performance; whereas for the other variables, it was set at 0.2 (Cohen's units). Suggested default probabilities to declare an effect clinically beneficial were $<0.5 \%$ for harm and $>25 \%$ for benefit. The effect was deemed unclear if it was possibly beneficial $(>25 \%)$ with an unacceptable risk of harm $(>0.5 \%)$. For mechanistic inferences, an effect was deemed unclear if the true value could be substantial in both a positive and a negative sense ( $>5 \%$ chance of being positive and negative). Where clear interpretation could be made, probabilities were assessed as presented by Hopkins et al. (11). The limits of agreement between the 100 - $\mathrm{m}$ time in the 2 conditions were derived according to the literature (6).

\section{Results}

\section{Baseline Measures}

Before warm-up, the physiological variables were not different between conditions. Baseline measurements of Tcore (control: $37.20 \pm 0.33^{\circ} \mathrm{C}$ vs. experimental: $37.29 \pm 0.44^{\circ} \mathrm{C} ; p=0.50$, $d=0.24)$, tympanic temperature $\left(36.73 \pm 0.83^{\circ} \mathrm{C}\right.$ vs. $36.76 \pm$ $\left.0.43^{\circ} \mathrm{C} ; p=0.87, d=0.05\right), \dot{\mathrm{V}}_{2}(5.59 \pm 0.85$ vs. $5.63 \pm 0.96$ $\left.\mathrm{ml} \cdot \mathrm{kg}^{-1} \cdot \mathrm{min}^{-1} ; p=0.90, d=0.04\right),\left[\mathrm{La}^{-}\right](2.88 \pm 0.78 \mathrm{vs}$. $\left.2.93 \pm 0.56 \mathrm{mmol} \cdot \mathrm{L}^{-1} ; p=0.85, d=0.06\right)$, salivary cortisol $\left(8.32 \pm 3.48\right.$ vs. $\left.9.58 \pm 3.68 \mathrm{nmol} \cdot \mathrm{L}^{-1} ; p=0.11, d=0.63\right)$, testosterone $\left(463.60 \pm 142.60\right.$ vs. $473.85 \pm 98.33 \mathrm{pmol} \cdot \mathrm{L}^{-1}$; $p=0.86, d=0.06)$, and testosterone/cortisol ratio (58.11 \pm 19.53 vs. $53.89 \pm 19.68 ; p=0.64, d=0.17$ ) were similar between the 2 conditions.

\section{Acute Response to Warm-up}

The acute responses to the different warm-ups are presented in Table 2. After the warm-up, there was a greater increase in 

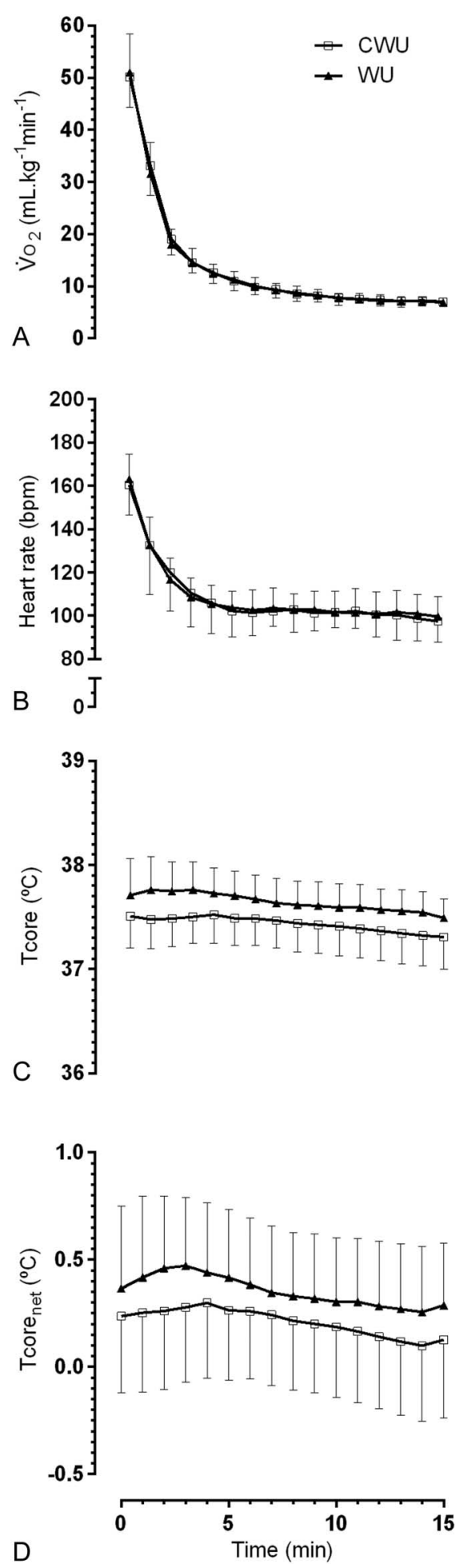

Figure 3. Comparison between the oxygen uptake $\left(\dot{\mathrm{V}}_{2}\right)(\mathrm{A})$, heart rate $(B)$, core temperature (C) (Tcore), and its net values (Tcore net ) (D) assessed during the 15 minutes of recovery after the $100 \mathrm{~m}$, with control warm-up (CWU) and experimental warm-up (WU). $n=13$.
$\dot{\mathrm{VO}}_{2}$, heart rate, and Tcore $_{\text {net }}$ in the experimental warm-up compared with control. Furthermore, the experimental condition was perceived by the swimmers to be very demanding. After the 10 minutes of rest that preceded the trial, the main differences had abated and there were unclear inferences among the physiological variables. However, the experimental warm-up condition still showed a positive effect on Tcore $_{\text {net }}$ by the time of the trial.

\section{Swim-Trial}

Table 3 presents the results recorded during the trial. Performance on the $100-\mathrm{m}$ time-trial was not different between conditions, with a "very likely" trivial effect. Additionally, Figure 2 illustrates the individual performance response to each of the warm-ups. Nine out of 13 swimmers performed better after the control warm-up and 4 after experimental warm-up.

The experimental warm-up led to decreased SF over the first $50 \mathrm{~m}$ and increased SL. Moreover, a positive effect on the $\eta_{\mathrm{p}}$ was found in the first lap after this warm-up. The main physiological acute adaptations to the maximal swimming test were found to be associated with $\left[\mathrm{La}^{-}\right]_{\text {peak }}$ and Tcore $_{\text {net }}$, which showed a medium negative or positive effect of experimental warm-up, respectively.

\section{Recovery Period}

Figure 3 depicts the physiological variables monitored over the recovery period, showing similar adaptations between the tested conditions. However, the Tcore net $_{\text {after }} 15$ minutes was moderately lower in the control warm-up compared with the experimental warm-up $(0.10 \pm 0.35$ vs. $0.26 \pm$ $\left.0.31^{\circ} \mathrm{C}, p=0.06, d=0.74\right)$. After the recovery period, $\left[\mathrm{La}^{-}\right](p=0.56, d=0.17)$, tympanic temperature $(p=0.42$, $d=0.23$ ) and salivary hormones (cortisol: $p=0.89, d=0.04$; testosterone: $p=0.49, d=0.20$; and testosterone/cortisol ratio: $p=0.91, d=0.03$ ) were not different between warmup conditions.

\section{Discussion}

The purpose of our study was to investigate the effects of 2 different warm-up intensities on maximal $100-\mathrm{m}$ freestyle time-trial performance. The results showed no differences in performance between a warm-up that included a race-pace set or one that included a set to increase $\dot{V}_{2}$. However, the warm-ups caused different acute physiologic adaptations. These differences abated during the time lag between warm-up and the time-trial, resulting in similar final $100-\mathrm{m}$ times. Despite similar performance outcomes, the biomechanical and physiological responses were different between trials. With the experimental condition, the efficiency was higher in the first lap and immediately after the race, Tcore $_{\text {net }}$ was higher, and $\left[\mathrm{La}^{-}\right]_{\text {peak }}$ was lower. In addition to physiological adaptations, our novel finding was the adaptation of the swimmers' technical pattern to match the preceding warmup. The different warm-ups seemed to trigger different race strategies to attain similar times, revealing the warm-up's 
importance in stimulating the physiological and biomechanical adjustments intended for the race.

The trivial differences in performance between conditions were lower than $0.1 \%$, confirming the findings of Houmard et al. (13). However, these authors did not assess maximal performance, and their conclusions were drawn based on the nonsignificant differences in heart rate, SL, and $\left[\mathrm{La}^{-}\right]$after a submaximal $365.8-\mathrm{m}$ freestyle. Interestingly, our results revealed different biomechanical patterns between conditions during the race. Neiva et al. (26) previously reported that warm-up exercises could influence biomechanics during maximal swimming. In the current study, the swimmers were able to achieve higher $\mathrm{SF}$ in the initial phase of the race after the control warm-up. In contrast, higher SL values were found after the experimental warm-up and a higher $\eta_{p}$ at the beginning of the race. It is accepted that swimmers are able to manipulate SL and SF to achieve a given velocity with the lowest energy cost (3). Consequently, those different biomechanical patterns could be what was most appropriate for the swimmers at each particular moment. Increased excitability of motor neurons by higher velocity improves the rate of force development and power production and may in this particular case have increased the SF after the control warm-up. The race-pace velocity was almost maximal, well above the critical velocity. Therefore, a different pattern could have been adopted because of differential stimulation (29). Hence, the warm-up can be used as an approach to activating a given biomechanical pattern.

Ajemian et al. (1) addressed an interesting finding on the relevance of motor learning during warm-up. Based on the assumption of humans' high learning rate for sensorimotor activity, the authors suggested that warm-up induces the recalibration of the sensorimotor network of athletes and restores their skills to a finely tuned state. This could justify the importance of warm-up specificity but also the different biomechanical patterns observed in this study. We infer that when the swimming velocity is increased in race-pace, the SF should increase (27). In contrast, SL should increase when the velocity decreases, as occurred in the experimental condition (27). Consequently, each condition acutely adapts the swimmers' motor skills according to the biomechanical pattern used and causes the swimmers to replicate these skills during the initial meters of the race.

The 2 warm-up procedures resulted in different acute physiological responses, with increased effort perceived after experimental warm-up; this could have influenced the way the swimmers raced in the time-trial. The higher Tcore $_{\text {net }}$ and $\dot{\mathrm{V}}_{2}$ observed immediately after warm-up in the experimental condition, with no increased $\left[\mathrm{La}^{-}\right]$, demonstrated that the main set succeeded in its goal of eliciting aerobic metabolism. According to the literature, one of the benefits of a warm-up is the increased $\mathrm{VO}_{2}$ at the beginning of the race, which contributes to improved performance $(5,20)$. The mechanisms for this increase are not clear, but some studies suggest that altered primary $\dot{\mathrm{V}}_{2}$ kinetics via a shorter time constant increases primary $\dot{\mathrm{V}}_{2}$ amplitude or/and reduces the $\mathrm{V}_{2}$ slow component (36). Despite the decrease in $\dot{\mathrm{V}}_{2}$ values during the 10 minutes of rest, and the lack of difference in $\dot{\mathrm{V}}_{2}$ and heart rate between conditions immediately before the trial, some of the initial effects could have been promoting internal adaptations.

The warm-up can change the metabolic profile of subsequent exercise by accelerating the $\dot{\mathrm{V}}_{2}$ kinetics and diminishing the blood lactate response (8). The increased $\mathrm{V}_{2}$ observed after experimental warm-up may have removed some of the inertia in mitochondrial activity (10). Accordingly, the aerobic system would have improved its preparedness state, allowing oxygen to be used at a faster rate at the beginning of the exercise and diminishing the reliance on anaerobic metabolism in this phase. Thus, a change in $\mathrm{VO}_{2}$ kinetics in the experimental condition could have allowed a faster response at the beginning, enabling a subsequently increased glycolytic contribution and explaining the similar $\dot{\mathrm{V}}_{2}$ peak and different $\left[\mathrm{La}^{-}\right]_{\text {peak }}$ after the trial. In addition, acceleration of overall $\mathrm{V}_{2}$ kinetics can occur because of enhanced oxygen delivery associated with increased blood flow to the muscles, which could in turn be associated with a rise in temperature (35). Our results showed higher Tcore $_{\text {net }}$ after warm-up and before the trial in the experimental condition.

During recovery, Tcore $_{\text {net }}$ was the only factor that retained a significant difference between conditions, with moderately higher values in the experimental condition. This increased temperature could be reflected in the "discomfort" felt by the swimmers, leading to a perception of the effort as "extremely hard" compared with the "hard" effort perceived for the control warm-up condition. The different perception could have even resulted from the increased $\mathrm{V}_{2}$ of the main set creating a greater imbalance in the homeostasis of the swimmers (31-33). It was expected that some of this impact on homeostasis would lead to hormonal variations between the conditions; changes in testosterone, cortisol, and its ratio levels could indicate anabolic or catabolic activity within the tissues $(15,17)$. Our findings correspond to the normal range of values for men reported in the literature $(12,15)$. However, no differences were observed between the 2 conditions, suggesting that the difference in warm-up intensity was not enough to shift the hormonal response.

Some limitations of the present study should be addressed. We acknowledge possible unknown variations in day-to-day performance because of daily events occurring outside the pool. Also, this study was performed for a specific race event; different distances or swimming techniques may elicit different adaptations, as may different ages and the use of female swimmers. Finally, an analysis of $\dot{\mathrm{V}}_{2}$ kinetics and muscle temperature could have improved our understanding of the mechanistic phenomenon.

In conclusion, the 2 swimming warm-up intensities resulted in no differences in performance in the $100-\mathrm{m}$ freestyle. Nevertheless, our findings have confirmed that varying warmup intensity results in the employment of different 
biomechanical strategies during the race and in different physiological/psychophysiological responses of the swimmers to each condition. Some physiological changes that occurred after the experimental warm-up were not present after control warm-up. The increased Tcore ${ }_{\text {net }}$ after warm-up until the end of the trial, the lower $\left[\mathrm{La}^{-}\right]_{\text {peak }}$ after the trial, and the increased swimming efficiency in the first meters of the race makes the use of an aerobic stimulation set during warmup a viable alternative to the usual warm-up comprising sets at higher swimming velocities. Yet, those differences were not reflected in the performance and require further investigation. In addition, our novel findings reveal the importance of warmup exercises with regard to sensorimotor adaptations to movement and motor skills. These should be performed at a level similar to the level intended for the race.

\section{Practical Applications}

Different warm-up intensities result in physiological and biomechanical changes during the race although the same performance results are obtained. The use of an aerobic stimulation set is a viable alternative to the traditional racepace set before the $100-\mathrm{m}$ freestyle. Moreover, there seems to be an acute learning process that could explain the different biomechanical patterns found during the race. If the race strategy depends on having a higher SF, a race-pace warm-up should be used, whereas if higher swimming efficiency is needed, aerobic stimulation should be used. Furthermore, an aerobic set increases core temperature and should be used when there is a long time-gap between the warm-up and the race.

\section{ACKNowledgments}

This project was supported by the National Funds through FCT-Portuguese Foundation for Science and Technology (UID/DTP/04045/2013)-and the European Fund for Regional Development (FEDER) allocated by European Union through the COMPETE 2020 Program (POCI-010145-FEDER-006969)-competitiveness and internationalization (POCI). This work was also supported by a grant from the Science and Technology Foundation (SFRH/ $\mathrm{BD} / 74950 / 2010)$ and by University of Beira Interior and Santander Totta Bank (UBI/FCSH/Santander/2010).

The authors have no conflicts of interest that are directly relevant to the content of this study. The results of the present study do not constitute endorsement of the product by the authors or the NSCA.

The authors disclose funding received for this work from any of the following organizations: National Institutes of Health (NIH); Welcome Trust; Howard Hughes Medical Institute (HHMI); and other(s).

\section{REFERENCES}

1. Ajemian, R, D'Ausilio, A, Moorman, H, and Bizzi, E. Why professional athletes need a prolonged period of warm-up and other peculiarities of human motor learning. J Mot Behav 42: 381-388, 2010.
2. Balilionis, G, Nepocatych, S, Ellis, CM, Richardson, MT, Neggers, $\mathrm{YH}$, and Bishop, PA. Effects of different types of warm-up on swimming performance, reaction time, and dive distance. J Strength Cond Res 26: 3297-3303, 2012.

3. Barbosa, TM, Bragada, JA, Reis, VM, Marinho, DA, Carvalho, C, and Silva, AJ. Energetics and biomechanics as determining factors of swimming performance: Updating the state of the art. J Sci Med Sport 13: 262-269, 2010.

4. Billat, LV. Interval training for performance: A scientific and empirical practice. Special recommendations for middle- and long-distance running. Part I: Aerobic interval training. Sports Med 31: 13-31, 2001.

5. Bishop, D. Warm up II: Performance changes following active warm up and how to structure the warm up. Sports Med 33: 483-498, 2003.

6. Bland, JM and Altman, DG. Applying the right statistics: Analyses of measurement studies. Ultrasound Obstet Gynecol 22: 85-93, 2003.

7. Borg, G. Borg's Perceived Exertion and Pain Scales. Champaign, IL: Human Kinetics, 1998.

8. Burnley, M, Doust, JH, and Jones, AM. Effects of prior warm-up regime on severe-intensity cycling performance. Med Sci Sports Exerc 37: 838-845, 2005.

9. Byrne, $\mathrm{C}$ and Lim, CL. The ingestible telemetric body core temperature sensor: A review of validity and exercise applications. Br J Sports Med 41: 126-133, 2006.

10. Campbell-O'Sullivan, SP, Constantin-Teodosiu, D, Peirce, N, and Greenhaff, PL. Low intensity exercise in humans accelerates mitochondrial ATP production and pulmonary oxygen kinetics during subsequent more intense exercise. J Physiol 538: 931-939, 2002.

11. Hopkins, W, Marshall, SW, Batterham, AM, and Hanin, J. Progressive statistics for studies in sports medicine and exercise science. Med Sci Sport Exerc 41: 3-13, 2009.

12. Hough, JP, Papacosta, E, Wraith, E, and Gleeson, M. Plasma and salivary steroid hormone responses of men to high-intensity cycling and resistance exercise. J Strength Cond Res 25: 23-31, 2011.

13. Houmard, JA, Johns, RA, Smith, LL, Well, JM, Kobe, RW, and McGoogan, SA. The effect of warm-up on responses to intense exercise. Int J Sports Med 12: 480-483, 1991.

14. Hughson, RL. Oxygen uptake kinetics: Historical perspective and future directions. Appl Physiol Nutr Metab 34: 840-850, 2009.

15. Jacks, DE, Savash, J, Anning, J, McGloughlin, T, and Andres, F. Effect of exercise at three exercise intensities on salivary cortisol. J Strength Cond Res 16: 286-289, 2002.

16. Jones, AM, Wilkerson, DP, Burnley, M, and Koppo, K. Prior heavy exercise enhances performance during subsequent perimaximal exercise. Med Sci Sports Exerc 35: 2085-2092, 2003.

17. Kochajska-Dziurowicz, A, Gawel-Szostek, V, Gabrys, T, and Kmita D. Changes in prolactin and testosterone levels induced by acute physical exertion in young female athletes. Hum Physiol 27: 349-352, 2001.

18. Laffite, LP, Vilas-Boas, JP, Demarle, A, Silva, J, Fernandes, R, and Billat, VL. Changes in physiological and stroke parameters during a maximal $400-\mathrm{m}$ free swimming test in elite swimmers. Can J Appl Physiol 29: S17-S31, 2004.

19. MacDonald, MJ, Naylor, HL, Tschakovsky, ME, and Hughson, RL. Peripheral circulatory factors limit rate of increase in muscle $\mathrm{O}_{2}$ uptake at onset of heavy exercise. J Appl Physiol 90: 83-89, 2001.

20. McGowan, CJ, Pyne, DB, Thompson, KG, and Rattray, B. Warm-up strategies for sport and exercise: Mechanisms and applications. Sports Med 45: 1523-1546, 2015.

21. McGowan, C, Rattray, B, Thompson, K, Pyne, D, and Raglin, J. Current warm-up practices and the contemporary issues faced by elite swimming coaches. J Sci Med Sport 18S: e82, 2014.

22. Mitchell, JB and Huston, JS. The effect of high- and low-intensity warm-up on the physiological responses to a standardized swim and tethered swimming performance. J Sports Sci 11: 159-165, 1993. 
23. Neiva, HP, Marques, MC, Barbosa, TM, Izquierdo, M, and Marinho, DA Warm-up and performance in competitive swimming. Sports Med 44: 319-330, 2014.

24. Neiva, HP, Marques, MC, Barbosa, TM, Izquierdo, M, Viana, JL, and Marinho, DA. Effects of $10 \mathrm{~min}$ vs. $20 \mathrm{~min}$ passive rest after warm-up on $100 \mathrm{~m}$ freestyle time-trial performance: A randomized crossover study. J Sci Med Sport 20: 81-86, 2017.

25. Neiva, HP, Marques, MC, Barbosa, TM, Izquierdo, M, Viana, JL, Teixeira, AM, and Marinho, DA. The effects of different warm-up volumes on the $100 \mathrm{~m}$ swimming performance: A randomized crossover study. J Strength Cond Res 29: 3023-3036, 2015.

26. Neiva, HP, Marques, MC, Fernandes, RJ, Viana, JL, Barbosa, TM, and Marinho, DA. Does warm-up have a beneficial effect on $100-\mathrm{m}$ freestyle? Int J Sports Physiol Perform 9: 145-150, 2014.

27. Pelayo, P, Sidney, M, Kherif, T, Chollet, D, and Tourny, C. Stroking characteristics in freestyle swimming and relationships with anthropometric characteristics. J Appl Biomech 12: 197-206, 1996.

28. Ribeiro, J, Figueiredo, P, Sousa, A, Monteiro, J, Pelarigo, J, Vila-Boas, JP, Toussaint, $\mathrm{HM}$, and Fernandes, $\mathrm{RF}$. $\mathrm{VO}_{2}$ kinetics and metabolic contributions during full and upper body extreme swimming intensity. Eur J Appl Physiol 115: 1117-1124, 2015.

29. Saez Saez de Villarreal, E, González-Badillo, JJ, and Izquierdo, M. Optimal warm-up stimuli of muscle activation to enhance short and long-term acute jumping performance. Eur J Appl Physiol 100: 393401, 2007.
30. Toubekis, AG and Tokmakidis, SP. Metabolic responses at various intensities relative to critical swimming velocity. J Strength Cond Res 27: 1731-1741, 2013.

31. van den Tillaar, R, Lerberg, E, and Von Heimberg, E. Comparison of three types of warm-up upon sprint ability in experienced soccer players. J Sport Health Sci, 2016. Epub ahead of print.

32. van den Tillaar, R, Vatten, T, and Von Heimburg, E. Effects of short or long warm-up on intermediate running performance. J Strength Cond Res 31: 37-44, 2017.

33. van den Tillaar, $\mathrm{R}$ and von Heimberg, E. Comparison of two types of warm-up upon repeated sprint performance in experienced soccer players. J Strength Cond Res 30: 2258-2265, 2016.

34. West, DJ, Dietzig, BM, Bracken, RM, Cunningham, DJ, Crewther, BT, Cook, CJ, and Kilduff, LP. Influence of post-warm-up recovery time on swim performance in international swimmers. J Sci Med Sport 16: 172-176, 2013

35. Willis, WT and Jackman, MR. Mitochondrial function during heavy exercise. Med Sci Sports Exerc 26: 1347-1353, 1994.

36. Wittekind, A and Beneke, R. Metabolic and performance effects of warm-up intensity on sprint cycling. Scand J Med Sci Sports 21: e201-e207, 2011.

37. Zochowski, T, Johnson, E, and Sleivert, GG. Effects of varying post warm-up recovery time on 200 -m time-trial swim performance. Int $J$ Sports Physiol Perform 2: 201-211, 2007. 